\title{
Lower Limit to the Scale of an Effective Quantum Theory of Gravitation
}

\author{
R. R. Caldwell ${ }^{1}$ and Daniel Grin ${ }^{2}$ \\ ${ }^{1}$ Department of Physics \& Astronomy, Dartmouth College, Hanover, New Hampshire 03755, USA \\ ${ }^{2}$ California Institute of Technology, Mail Code 130-33, Pasadena, California 91125, USA \\ (Received 6 June 2006; revised manuscript received 19 September 2007; published 22 January 2008) \\ An effective quantum theory of gravitation in which gravity weakens at energies higher than $\sim 10^{-3} \mathrm{eV}$ \\ is one way to accommodate the apparent smallness of the cosmological constant. Such a theory predicts \\ departures from the Newtonian inverse-square force law on distances below $\sim 0.05 \mathrm{~mm}$. However, it is \\ shown that this modification also leads to changes in the long-range behavior of gravity and is inconsistent \\ with observed gravitational lenses.
}

DOI: 10.1103/PhysRevLett.100.031301

PACS numbers: 04.60. $-\mathrm{m}, 04.50 .-\mathrm{h}, 04.80 . \mathrm{Cc}$

The discovery of the cosmic acceleration [1] has prompted speculations of new physics. A leading hypothesis is the existence of a cosmological constant, responsible for the accelerated expansion. The milli-eV energy scale implied by this phenomenon is difficult to understand in terms of a fundamental theory [2]. The validity of Einstein's general theory of relativity (GR) on cosmological scales has thus come under suspicion. A novel solution to this problem might be achieved if GR is a low-energy effective theory in which gravity weakens at some energy scale. In an effective theory of gravity there may exist a threshold, $\mu$, beyond which gravitons cannot mediate momentum transfers. This behavior may be due to a "fat" graviton, a minimal length scale associated with quantum gravity, or possibly nonlinear effects which filter out highfrequency interactions [3-10]. Such theories offer a novel solution to the cosmological constant problem by regulating the contribution of vacuum fluctuations to the cosmological constant. However, we show that this mechanism may have already been explored and ruled out by gravitational lensing on cosmological scales.

We estimate the energy scale of an effective theory of gravitation by matching the predicted quantum vacuum energy density with the energy density of a cosmological constant, $\Lambda$, necessary to explain the accelerated cosmic expansion. Following Zeldovich [11], the gravitating energy density of the particle physics vacuum as due to $N$ equivalent, massless scalar particles, is

$$
\rho_{\Lambda}=\frac{N}{2} \int \frac{d^{3} k}{(2 \pi \hbar)^{3}} k c f(k) .
$$

We introduce the function $f(k)=e^{-k / \mu}$ to regulate the momentum at the vertex where vacuum bubbles connect to gravitons in order to limit the gravitating energy density. We refer to $\mu$ as a "cutoff" scale in the sense that the standard gravitational interactions are severely weakened above this scale. We match $\rho_{\Lambda}=\Omega_{\Lambda} \rho_{\text {crit }}$ and obtain $\mu=$ $0.0048\left(\Omega_{\Lambda} h^{2} / N\right)^{1 / 4} \mathrm{eV} / c$ as the desired cutoff scale. Current measurements give $\Omega_{\Lambda} h^{2}=0.34 \pm 0.04(1 \sigma)$ (see Ref. [12] and references therein) so that $\mu=$
$0.0037(1 \pm 0.03) / N^{1 / 4} \mathrm{eV} / c$. We now examine the consequences of this cutoff.

We consider weak gravitational fields described by a linearized, effective quantum theory of gravity [13]. The interaction Lagrangian at lowest order is

$$
\mathcal{L}_{I}=-\frac{1}{2} \kappa h_{\mu \nu} T^{\mu \nu},
$$

where $\kappa=\sqrt{32} \bar{\pi} \bar{G}, h_{\mu \nu}$ is the graviton field, and $T^{\mu \nu}$ is the stress-energy tensor of the gravitating sources. Here, we introduce an exponential cutoff at $\mu$ on graviton momenta.

Short-distance gravitational phenomena below the length $\ell_{0}=\hbar / \mu \sim 0.05 \mathrm{~mm}$ are affected by such a cutoff, which we impose on the graviton four-momentum $q^{\mu}$ so that $q^{2} \equiv q^{\mu} q_{\mu}<\mu^{2}$. For real gravitons, $q^{\mu} q_{\mu}=0$ and so the constraint is trivially satisfied. For virtual gravitons, the cutoff may be imposed by suppressing the graviton propagator in the ultraviolet [14]: $1 / q^{2} \rightarrow G\left(q^{2} / \mu^{2}\right) / q^{2}$, where $G$ is a function of the graviton momentum. For example, our exponential cutoff follows if $G(x)=e^{-\sqrt{x}}$. Such a modified propagator follows naturally from modified gravitational Lagrangians. This is clear upon inspection of the weak-field, Coulomb gauge, gravitational Lagrangian for a "fading gravity" model [14]:

$$
\mathcal{L}_{g}=2\left(h^{\alpha \beta}-\frac{1}{2} \eta^{\alpha \beta} h\right) \mathcal{G}^{-1}\left(\square / \mu^{2}\right) \square h_{\alpha \beta},
$$

where $\square$ is the D'Alembertian operator. The sum of (2) and (3) can be used to obtain the weak-field equations of motion.

An exponential cutoff to the momentum-space integral for the virtual gravitons exchanged between two static masses, $m_{1}$ and $m_{2}$, changes the Newtonian potential to

$$
\begin{aligned}
V & =-8 \pi G m_{1} m_{2} \int \frac{d^{3} q}{(2 \pi)^{3} \hbar} \frac{1}{2 q^{2}} e^{(i / \hbar) \vec{q} \cdot\left(\vec{x}_{1}-\vec{x}_{2}\right)} f(q) \\
& =-\frac{G m_{1} m_{2}}{r} \frac{2}{\pi} \arctan \frac{r}{\ell_{0}} .
\end{aligned}
$$

Relativistic corrections to the potential are similarly modi- 
fied $[15,16]$. The above expression asymptotes to the standard result for $r \gg \ell_{0}$ but reaches a finite minimum as $r / \ell_{0} \rightarrow 0$. Hence, static masses become free of gravitation at short distances.

The possibility of new gravitational phenomena at submillimeter distances has motivated laboratory tests of the Newtonian force law [17-22]. These experiments look for departures from the Newtonian force law, which are interpreted as bounds on a Yukawa-type modification of the potential, $V=-\frac{G m_{1} m_{2}}{r} \times\left(1+\alpha e^{-r / \lambda}\right)$. The potential (4) roughly corresponds to $\alpha \sim-1$ and $\lambda \sim \ell_{0}$. Recent measurements show that the Newtonian force law holds down to $56 \mu \mathrm{m}$ for $|\alpha|=1$ so that $\mu>0.0035 \mathrm{eV} / c$ at the $95 \%$ confidence level [22]. These efforts are at the threshold of the scale inferred from $\Lambda$.

Long-distance gravitational phenomena are also sensitive to such modifications and provide a tighter bound on $\mu$, the scale of new physics. The key is the limited range of graviton momenta mediating the gravitational force exerted by a massive body on a test particle. Considering the deflection of light as an elastic, quantum mechanical scattering process, the photon energy is conserved, but its momentum is redirected. A maximum graviton momentum implies a maximum deflection angle, and so $\left|\vec{k}_{\gamma i}-\vec{k}_{\gamma f}\right| \approx$ $2 k_{\gamma} \theta<\mu$, where $k_{\gamma}$ is the photon momentum.

We perform a calculation of tree-level photon scattering in linearized quantum gravity. We treat the lens as one massive particle, as many constituent particles, or as the source of an external gravitational field. All approaches yield the same result. The external field offers the clearest view. The cross section is

$$
\sigma=(2 \pi)^{2} \int d^{3} k_{\gamma f} \delta\left(k_{\gamma i}-k_{\gamma f}\right)\left|\left\langle k_{\gamma f}|\mathcal{M}| k_{\gamma i}\right\rangle\right|^{2}
$$

for a given photon polarization. The Maxwell tensor $T^{\mu \nu}=F^{\mu \rho} F_{\rho}^{\nu}-\frac{1}{4} \eta^{\mu \nu} F_{\alpha \beta} F^{\alpha \beta}$ is used in (2) to determine the scattering vertex, and the matrix element is calculated in the external-field approximation, using $h^{\mu \nu}$ for a weak gravitational field due to a point source of mass $M$. Following Refs. [23,24] we obtain

$$
\begin{aligned}
\left\langle k_{\gamma f}|\mathcal{M}| k_{\gamma i}\right\rangle= & \frac{8 \pi G M}{2(2 \pi)^{2}} \sqrt{k_{\gamma f} k_{\gamma i}} \frac{e^{-\left|\vec{k}_{\gamma f}-\vec{k}_{\gamma i}\right| / \mu}}{\left|\vec{k}_{\gamma f}-\vec{k}_{\gamma i}\right|^{2}} \Pi(e, k) \\
\Pi(e, k)= & \frac{1}{\sqrt{2}}\left[\left(\hat{e}_{i} \cdot \hat{e}_{f}^{*}\right)\left(3-\hat{k}_{\gamma i} \cdot \hat{k}_{\gamma i}\right)\right. \\
& \left.+\left(\hat{e}_{f}^{*} \cdot \hat{k}_{\gamma i}\right)\left(\hat{e}_{i} \cdot \hat{k}_{\gamma f}\right)\right],
\end{aligned}
$$

where $\hat{e}$ is the photon polarization vector. Averaging over incoming photon polarizations and summing over outgoing polarizations, we obtain the differential cross-section in the small angle limit

$$
\frac{d \sigma}{d \Omega}=\frac{(4 G M)^{2}}{(c \theta)^{4}} e^{-2 \theta k_{\gamma} / \mu} .
$$

In the absence of the cutoff, the cross section has the familiar $\theta^{-4}$ dependence found in Coulomb scattering. With the cutoff, we interpret the result to indicate that high-energy photons find a weaker gravitational lens than low-energy photons. This stands in contrast with the achromatic nature of lensing in general relativity.

It is not surprising that gravitational lensing can be described by a tree-level diagram. As with Coulomb scattering, a tree-level diagram is sufficient to reproduce the classical result. We may also calculate the contribution of higher-order Feynman diagrams in the eikonal limit, wherein the total energy of the colliding particles vastly exceeds the momentum transfer. This applies to astrophysical gravitational lensing. In perturbative quantum gravity, graviton loop diagrams are responsible for the nonrenormalizability of the theory and lead to a loss of predictive power at high energies. In the eikonal limit, these diagrams are negligible compared to the series of ladder and crossedladder diagrams illustrated in Fig. 1. As shown in Refs. [25,26], the amplitude for gravitational scattering of two massive scalar particles can then be summed to all orders in perturbation theory. In the absence of a cutoff on graviton momenta, this procedure yields the amplitude multiplied by a divergent phase factor. Since the cross section depends on $|\mathcal{M}|^{2}$, the Born approximation for the cross section is exact. We generalize this result to the case with the cutoff. We work in the rest frame of the massive scatterer and include an exponential factor for the momentum cutoff on each graviton propagator. The photon is adequately treated as a massless scalar in the limit of small deflections. Then, following Ref. [26], the scattering amplitude due to an infinite sum of ladder graphs in the eikonal limit is

$$
\begin{aligned}
i \mathcal{M}= & \frac{8 \pi M E_{\gamma}}{q^{2}} e^{-q / \mu} \int_{0}^{\infty} d z z J_{0}(z)\left\{\left[k_{\mathrm{IR}} / \mu\right.\right. \\
& \left.\left.+\sqrt{\left(k_{\mathrm{IR}} / \mu\right)^{2}+\left(z k_{\mathrm{IR}} / q\right)^{2}}\right]^{4 i \eta}-1\right\} .
\end{aligned}
$$

As in QED, the infrared regulator $k_{\mathrm{IR}}$ is necessary because the asymptotic states assumed were plane waves, rather than Coulombic wave functions. To proceed, we make a series expansion in small $k_{\mathrm{IR}} / \mu$. Then, because $\eta \equiv$ $G M E_{\gamma} \gg 1$, the integral is found to be well approximated by

$$
i \mathcal{M}=i \mathcal{M}_{\mathrm{Born}, \mathrm{GR}} e^{-q / \mu}\left(\frac{4 k_{\mathrm{IR}}^{2}}{q^{2}}\right)^{2 i \eta} \frac{\Gamma(1+2 i \eta)}{\Gamma(1-2 i \eta)} e^{i q / \mu},
$$

where $\mathcal{M}_{\mathrm{Born}, \mathrm{GR}}=32 \pi G M^{2} E_{\gamma}^{2} / q^{2}$ for the gravitational scattering of these two scalar particles. This nonperturbative result consists of the exponentially suppressed Born amplitude with an additional phase which does not affect the scattering cross section. Thus our tree-level result is exact in the eikonal limit.

As opposed to multiple graviton exchange in a single scattering interaction, we may also consider multiple encounters along the particle trajectory. For photons impinging on a target with an impact parameter $b$, the gravi- 
a)

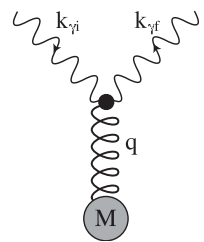

b)

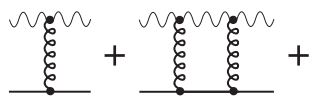

FIG. 1. (a) The Feynman diagram for the gravitational deflection of light. (b) The leading ladder and crossed-ladder Feynman diagrams for graviton exchange are shown.

tational interaction time is $\sim b / c$. In comparison, the interval during which the photon is in the near, scattering zone of the gravitational lens is $\Delta t \sim b / c$. From the similarity of these time scales, we expect that the photon will experience but a single scattering interaction. For a nonrelativistic particle of velocity $v$, we expect the interval $\Delta t \sim b / v$ will be much greater than $b / c$. We thus expect the deflection to be determined by many, successive singlegraviton exchange interactions with the central mass. Hence, bound systems as well as the scattering of massive objects, such as satellites or stars, are insensitive to the cutoff since they exchange lower momentum gravitons at each vertex.

We can also consider the photon deflection as arising from multiple scattering events off the constituent particles in the deflector mass. In QED, when an electron scatters off a heavy nucleus, it has a single photon vertex, but each charged nucleon couples coherently to a virtual photon. The total scattering matrix element is the sum of the matrix elements due to the individual scatterers [27]. If $\mathcal{M}_{j}$ is the matrix element for the $j$ th scatterer, the total amplitude is $\left|\mathcal{M}_{\text {tot }}\right|^{2}=\sum_{j}\left|\mathcal{M}_{j}\right|^{2}+\sum_{j \neq j^{\prime}} \mathcal{M}_{j}^{*} \mathcal{M}_{j^{\prime}}$. For $Z$ constituent particles there are $Z$ diagonal terms and $Z(Z-1)$ offdiagonal terms. Evaluation of the off-diagonal terms requires the correlations between $j, j^{\prime}$ pairs of particles. The incoming electron scatters coherently, as is the case for weak deflections in which the internal momenta of the nucleons are negligible, so the $j$ particles all move with the nucleus zero mode, and the correlations are effectively delta functions. Upon integration over the phase space to obtain the differential cross section, the $Z^{2}$ diagonal and off-diagonal terms contribute equally, and so the multiple scattering approach yields the same result as scattering off the collective nucleus.

In the case of gravitational deflection, we may consider the deflector mass $M$ as consisting of $Z$ smaller objects of mass $M / Z$, which includes the gravitational binding energy. For typical gravitational lens systems, the impact parameter is much greater than the de Broglie wavelength corresponding to the total momentum transfer. Thus, we are in the limit of coherent scattering, and as in QED, the same result is obtained whether we employ the point particle or multiple scattering description. Since the scattered particle has only one vertex, the cutoff leads to the same constraint on the change in photon momentum, resulting in Eq. (7) for the cross section.
To interpret the cross section in terms of a deflection angle, we consider an incident beam of light at impact parameter $b$. The beam is deflected into an area $d \sigma=b d b d \phi$, which gives us a differential relating $\theta$ and $b$. For small angles, this differential can be integrated to yield $4 G M /\left(b c^{2}\right)=\theta / F\left(2 \theta k_{\gamma} / \mu\right)$, where $F(x)=$ $\sqrt{(1-x) e^{-x}-x^{2} \operatorname{Ei}(-x)}$ and $\operatorname{Ei}(x) \equiv-\int_{-x}^{\infty} e^{-t} d t / t$ is the exponential-integral function. Defining $\theta_{\mathrm{GR}} \equiv$ $4 G M /\left(b c^{2}\right)$ for the standard result without the cutoff, then $\theta / \theta_{\mathrm{GR}}=F\left(2 \theta k_{\gamma} / \mu\right)$. We note that the static, frequency-independent metric potential is insufficient to describe the photon's path past the lensing source when $\theta_{\mathrm{GR}} \geq \mu / 2 k_{\gamma}$. It would be necessary to introduce an effective force into the geodesic equation, based on the modified graviton propagator. We thus find that the deflection is half the standard prediction when $2 \theta k_{\gamma} / \mu \sim 1$. In the limit $\theta \ll \mu / 2 k_{\gamma}, F \rightarrow 1$, but for $\theta \gtrsim \mu / 2 k_{\gamma}$ the deflection angle is suppressed. Hence, we would expect a dearth of gravitationally lensed images of high-frequency light if there were a cutoff in graviton momentum.

Numerous gravitational lens systems have been observed from radio to $\mathrm{x}$-ray frequencies. The tightest constraint to $\mu$ comes from $\mathrm{x}$-ray observations of the gravitationally lensed system Q0957 + 561 [28]. For this lens system, image A due to the quasar at $z=1.4$ appears $5.2^{\prime \prime}$ away from the primary lensing galaxy at $z=0.36$ [29]. Using the angular-diameter distances to the source and from lens to the source, $D_{S}, D_{\mathrm{LS}}$, to reconstruct the lensing geometry, we estimate a deflection angle of $\theta=$ $5.2^{\prime \prime} \times D_{S} / D_{\mathrm{LS}}=7.8^{\prime \prime}$. The lens image locations are unchanged for $E_{\gamma}<5 \mathrm{keV}$ [30], which yields the lower bound $\mu>0.38 \mathrm{eV} / c$. This result pushes the threshold for departures from the Newtonian force law down to $0.5 \mu \mathrm{m}$.

This lower limit is nearly 2 orders of magnitude higher than, and therefore rules out, the cutoff inspired by the cosmological constant with $N>1$. If $N \ll 1$ perhaps due to a cancellation of bosonic and fermionic contributions, then agreement is still possible. We have also tried other forms for the cutoff, including a Gaussian and a sharp power law and find that our results do not change appreciably. This bound may also constrain dark energy models, where such a cutoff prevents the spontaneous decay of the vacuum into phantom or ghost particles [31-34]. We caution the reader that our results apply only to effective theories in which gravity weakens above the cutoff scale in a way described by the implementation of the cutoff function $f(q)$. A tighter constraint may be obtained in the future from hard x-ray or gamma-ray observations of lens images.

It is instructive to compare our graviton momentum cutoff with a similar cutoff in the electron-phonon interaction. In metals, the phonon plays an important role in the dynamics of conduction electrons, conveying an attractive long-range interaction between electrons, which partially cancels the Coulomb interaction. The phonon has an ef- 
fective width or frequency which characterizes the response time of the ion lattice, above which the phonon interaction is suppressed. The bare pseudopotential extracted from the electron-phonon matrix element must be dressed by frequency-dependent factors which include the limited phonon response, in order to produce an accurate picture of the electron dynamics (e.g., [35]). By analogy with the phonon, we expect the effective width of the graviton to lead to a dramatic change in the behavior of gravitational scattering, shifting the boundary between classical and quantum gravitational interactions. Tree-level amplitudes, which are usually regarded as classical due to the absence of any $\hbar$ factor, are quantum corrected by the presence of the phenomenological scale $\mu$. We expect that the static gravitational potential will be of limited use, since it may not fully capture the effects of the limited graviton response on kinematics.

We note that a graviton cutoff would lead to a suppression of the spectrum of inflationary gravitational waves. The highest frequency graviton modes allowable by the cutoff enter the horizon when $H \sim c \mu / \hbar$, at which time the cosmic temperature is $\sim 2 \mathrm{TeV}$ for a cutoff based on the magnitude of $\Lambda$. These waves redshift down to a frequency $\sim 2 \times 10^{-4} \mathrm{~Hz}$ by the present day. Hence, there would be no inflationary gravitational waves in the frequency range of the proposed Big Bang Observer [36] satellite gravitational wave detector.

We have explored the consequences of a simplistic treatment of the cosmological constant problem. Here, with the introduction of the momentum scale $\mu$, the classical regime is restricted to soft interactions with low momentum transfers; hard scattering must take into account the suppression factor on the graviton propagator. One may expect a cutoff to play some role in separating the high-energy and low-energy domains of the underlying, fundamental theory of gravity. At energy scales above the cutoff, gravity may weaken and then lensing imposes an important bound.

We thank George Chartas for sharing his Chandra results. We thank the TAPIR group and Mark Wise at Caltech for useful discussions. R. C. was supported in part by NSF No. AST-0349213 and Research Corporation RI-0887 at Dartmouth.

[1] A. G. Riess et al., Astron. J. 116, 1009 (1998); B. P. Schmidt et al., Astrophys. J. 507, 46 (1998); P. M. Garnavich et al., Astrophys. J. 509, 74 (1998); S. Perlmutter et al., Astrophys. J. 517, 565 (1999).

[2] S. Weinberg, Rev. Mod. Phys. 61, 1 (1989); S. M. Carroll, Living Rev. Relativity 4, 1 (2001); P. J.E. Peebles and B. Ratra, Rev. Mod. Phys. 75, 559 (2003); T. Padmanabhan, Phys. Rep. 380, 235 (2003).
[3] A. Zee, Phys. Lett. B 594, 8 (2004).

[4] R. Sundrum, Phys. Rev. D 69, 044014 (2004).

[5] R. Sundrum, Nucl. Phys. B690, 302 (2004).

[6] S. Hossenfelder, Phys. Rev. D 73, 105013 (2006).

[7] T. Padmanabhan, Phys. Rev. Lett. 78, 1854 (1997).

[8] M. Reuter and J. M. Schwindt, J. High Energy Phys. 01 (2007) 049.

[9] M. Reuter and J. M. Schwindt, J. High Energy Phys. 01 (2006) 070.

[10] G. Dvali, S. Hofmann, and J. Khoury, arXiv:hep-th/ 0703027.

[11] Y. B. Zel'dovich, Zh. Eksp. Teor. Fiz. 6, 883 (1967) [JETP Lett. 6, 316 (1967)].

[12] M. Roos, arXiv:astro-ph/0509089.

[13] R. P. Feynman, Acta Phys. Pol. 24, 697 (1963); B.S. DeWitt, Phys. Rev. 160, 1113 (1967); M. J. G. Veltman, in Methods in Field Theory, Proceedings of the Les Houches Summer School, Session XXVIII, edited by R. Balian and J. Zinn-Justin (North-Holland, Amsterdam, 1976); G. Papini and S. R. Valluri, Phys. Rep. 33, 51 (1977); J.F. Donoghue, Phys. Rev. D 50, 3874 (1994).

[14] J. Khoury, arXiv:hep-th/0612052.

[15] E. Corinaldesi, Proc. Phys. Soc. London Sect. A 69, 189 (1956).

[16] B. Barker, S. Gupta, and R. Haracz, Phys. Rev. 149, 1027 (1966).

[17] C. D. Hoyle et al., Phys. Rev. Lett. 86, 1418 (2001).

[18] J. Chiaverini et al., Phys. Rev. Lett. 90, 151101 (2003).

[19] J. C. Long et al., Nature (London) 421, 922 (2003).

[20] C. D. Hoyle et al., Phys. Rev. D 70, 042004 (2004).

[21] S. J. Smullin et al., Phys. Rev. D 72, 122001 (2005); 72, 129901(E) (2005).

[22] D. J. Kapner et al., Phys. Rev. Lett. 98, 021101 (2007).

[23] D. Boccaletti et al., Nuovo Cimento A 48, 58 (1967).

[24] W. K. De Logi and S. J. Kovacs, Phys. Rev. D 16, 237 (1977).

[25] G. 't Hooft, Phys. Lett. B 198, 61 (1987).

[26] D. Kabat and M. Ortiz, Nucl. Phys. B388, 570 (1992).

[27] M. Lax, Rev. Mod. Phys. 23, 287 (1951).

[28] G. Chartas et al., Astrophys. J. 565, 96 (2002).

[29] See http://www.cfa.harvard.edu/castles.

[30] G. Chartas (private communication).

[31] S. M. Carroll, M. Hoffman, and M. Trodden, Phys. Rev. D 68, 023509 (2003).

[32] J. M. Cline, S. Jeon, and G. D. Moore, Phys. Rev. D 70, 043543 (2004).

[33] S. D. H. Hsu, A. Jenkins, and M. B. Wise, Phys. Lett. B 597, 270 (2004).

[34] D. E. Kaplan and R. Sundrum, J. High Energy Phys. 07 (2006) 042

[35] D. J. Scalapino, in Superconductivity, edited by R.D. Parks (Marcel Dekker, Inc., New York, 1969).

[36] S. Phinney et al., "The Big Bang Observer: Direct Detection of Gravitational Waves from the Birth of the Universe to the Present, NASA Mission Concept Study," 2004. 